\title{
THE RESONANCE COUNTING FUNCTION FOR SCHRÖDINGER OPERATORS WITH GENERIC POTENTIALS
}

\author{
T. Christiansen And P. D. Hislop
}

\begin{abstract}
We show that the resonance counting function for a Schrödinger operator has maximal order of growth for generic sets of real-valued, or complex-valued, $L^{\infty}$-compactly supported potentials.
\end{abstract}

\section{Introduction}

The purpose of this note is to show that for a generic set of compactly supported potentials, the resonance counting function for the associated Schrödinger operator has maximal order of growth. We consider odd dimensions $d$, and any potential $V \in L_{\text {comp }}^{\infty}\left(\mathbb{R}^{d}\right)$. We define the set of scattering poles or resonances of the Schrödinger operator $H_{V} \equiv-\Delta+V$ on $L^{2}\left(\mathbb{R}^{d}\right)$ through the meromorphic continuation of the resolvent. To make this precise, let $\chi_{V}$ be a smooth, compactly supported function equal to one on the support of $V$. It is well-known that the operator-valued function $\lambda \rightarrow \chi_{V}\left(H_{V}-\lambda^{2}\right)^{-1} \chi_{V}$ admits a meromorphic continuation (denoted by the same symbol) from $\operatorname{Im} \lambda \geq 0$, taken as the physical half-plane, to the entire complex plane. The poles of this continuation (including multiplicities) are independent of the choice of $\chi_{V}$ satisfying these conditions. There are at most a finite number of poles with $\operatorname{Im} \lambda>0$ corresponding to the finitely-many eigenvalues of $H_{V}$. The set of scattering poles of $H_{V}$ is defined by

$$
\begin{aligned}
\mathcal{R}_{V}= & \left\{\lambda_{j} \in \mathbb{C}: \chi_{V}\left(H_{V}-\lambda^{2}\right)^{-1} \chi_{V}\right. \\
& \text { has a pole at } \left.\lambda=\lambda_{j}, \text { listed with multiplicity }\right\}
\end{aligned}
$$

This definition can be made for both real-valued and complex-valued potentials. The resonance counting function $N_{V}(r)$ for $H_{V}$ on $L^{2}\left(\mathbb{R}^{d}\right)$, is defined as

$$
N_{V}(r)=\#\left\{\lambda_{j} \in \mathcal{R}_{V}:\left|\lambda_{j}\right|<r\right\} .
$$

The large $r$ properties of $N_{V}(r)$ have been extensively studied, and we refer the reader to the review article of Zworski [19]. The leading asymptotic behavior in one dimension was proved by Zworski [22] (see also [3, 12]) and for certain spherically symmetric potentials for odd $d \geq 3[20]$. Moreover, the following

Received by the editors May 23, 2005.

TC partially supported by NSF grant DMS 0088922 and PDH partially supported by NSF grant DMS 0202656. 
upper bound on $N_{V}(r)$ for compactly supported potentials, due to Zworski [21], is now well-known

$$
N_{V}(r) \leq C_{V, d}\left(1+r^{d}\right)
$$

see $[4,5,14,15,20]$ for related results and other proofs. In addition, for nontrivial real-valued, compactly supported potentials, it is known that an infinite number of resonances exist $[6,9,10]$. More recently, Sá Barreto [8] proved a lower bound of the form

$$
\lim \sup _{r \rightarrow \infty} \frac{N_{V}(r)}{r}>0
$$

for nontrivial $V \in C_{c}^{\infty}\left(\mathbb{R}^{d} ; \mathbb{R}\right)$. The situation is different for complex-valued, $L^{\infty}$ compactly supported potentials. There are nontrivial examples of such potentials with no resonances for $d \geq 3[1]$.

The purpose of this note is to prove that the resonance counting function $N_{V}(r)$, defined in (2), has the maximal order of growth $d$ for a generic family of either real-, or complex-valued, compactly supported potentials. Following B. Simon [11], for a metric space $X$, we call a dense $G_{\delta}$ set $S \subset X$ Baire typical. Our main result is the following theorem.

Theorem 1.1. Let $d \geq 3$ be odd, let $K \subset \mathbb{R}^{d}$ be a compact set with nonempty interior, and let $F=\mathbb{R}$ or $F=\mathbb{C}$. Then the set

$$
\mathcal{M}=\left\{V \in L^{\infty}(K ; F): \lim \sup _{r \rightarrow \infty} \frac{\log N_{V}(r)}{\log r}=d\right\}
$$

is Baire typical in $L^{\infty}(K ; F)$.

We remark that a similar result for potentials in $C^{\infty}(K ; F)$ is true in the $C^{\infty}$ topology. The proof is very similar to that given here, but requires the use of [2, Theorem 1.2] in place of [20, Theorem 2].

The term generic is often used to to describe a set which is the intersection of a countable number of open dense sets [16]. If $X$ is a perfect complete metric space and $A \subset X$ is such a generic set, then for any open ball $B_{X} \subset X$ the intersection $A \cap B_{X}$ is uncountable. In this sense, our theorem says that the resonance counting function for a generic set of real- or complex-valued, $L^{\infty}$ compactly supported potentials has the maximum order of growth given by the dimension $d \geq 1$ and odd. Since there are nontrivial, complex-valued, $L^{\infty}$ compactly supported potentials for which $N_{V}(r)$ has zero order of growth [1], and since $N_{0}(r)$ for the Laplacian (zero real potential) has zero order of growth, our result is the best possible. We remark that it would be interesting to find nontrivial potentials $V \in L_{\text {comp }}^{\infty}\left(\mathbb{R}^{d} ; \mathbb{R}\right), d \geq 3$ and odd, for which the order of growth of $N_{V}(r)$ is strictly less than $d$.

\section{Proof of Theorem 1.1}

We shall denote the scattering matrix for $H_{V}=-\Delta+V$ by $S_{V}(\lambda)$. The operator $S_{V}(\lambda)$ acts on $L^{2}\left(S^{d-1}\right)$ and if $V$ is real-valued, then it is a unitary 
operator for $\lambda \in \mathbb{R}$. The $S$-matrix is given explicitly by

$$
S_{V}(\lambda)=I+c_{d} \lambda^{d-2} \pi_{\lambda}\left(V-V R_{V}(\lambda) V\right) \pi_{-\lambda}^{t} \equiv I+T_{\lambda},
$$

where $R_{V}(\lambda)=\left(H_{V}-\lambda^{2}\right)^{-1}$ and $\left(\pi_{\lambda} f\right)(\omega)=\int e^{-i \lambda x \cdot \omega} f(x) d x$ [17]. Under the assumption that $V \in L_{\text {comp }}^{\infty}\left(\mathbb{R}^{d} ; F\right)$, the operator $T_{\lambda}: L^{2}\left(S^{d-1}\right) \rightarrow L^{2}\left(S^{d-1}\right)$ is trace class. The $S$-matrix has a meromorphic continuation to the entire complex plane with finitely many poles in $\operatorname{Im} \lambda>0$, corresponding to eigenvalues of $H_{V}$, and resonances in $\operatorname{Im} \lambda<0$. We recall that if $\operatorname{Im} \lambda_{0} \geq 2\|V\|_{L^{\infty}}+1$, the multiplicities of $\lambda_{0}$, as a zero of $\operatorname{det} S_{V}(\lambda)$, and $-\lambda_{0}$, as a pole of $\left(H_{V}-\lambda^{2}\right)^{-1}$, coincide, cf. Section 3 of [18]. We will work with the function $\operatorname{det} S_{V}(\lambda)$. For $N, M, q>0, j>2 N+1$, let

$$
\begin{array}{r}
A(N, M, q, j)=\left\{V \in L^{\infty}(K ; F):\|V\|_{L^{\infty}} \leq N, \log \left|\operatorname{det}\left(S_{V}(\lambda)\right)\right| \leq M|\lambda|^{q}\right. \\
\text { for } \operatorname{Im} \lambda \geq 2 N+1 \text { and }|\lambda| \leq j\} .
\end{array}
$$

We remark that $\operatorname{det} S_{V}(\lambda)$ is holomorphic in this region.

Lemma 2.1. The set $A(N, M, q, j) \subset L^{\infty}\left(\mathbb{R}^{d}\right)$ is closed.

Proof. Let $V_{k} \in A(N, M, q, j)$, such that $V_{k} \rightarrow V$ in the $L^{\infty}$ norm. Then clearly $\|V\|_{L^{\infty}} \leq N$. We shall use (5) and the bound

$$
|\operatorname{det}(I+A)-\operatorname{det}(I+B)| \leq\|A-B\|_{1} e^{\|A\|_{1}+\|B\|_{1}+1},
$$

cf. [13]. We let $\|\cdot\|_{1}$ and $\|\cdot\|_{2}$ denote the trace and Hilbert-Schmidt norms. We wish to show that $\left\|S_{V_{k}}(\lambda)-S_{V}(\lambda)\right\|_{1} \rightarrow 0$ as $k \rightarrow \infty$. Let $\chi \in C_{c}^{\infty}\left(\mathbb{R}^{d}\right)$ be a function that is equal to one on $K$. Using (5), we have

$$
\begin{aligned}
& \left\|S_{V_{k}}(\lambda)-S_{V}(\lambda)\right\|_{1} \\
& \quad \leq\left|c_{d}\left\|\left.\lambda\right|^{d-2}\right\| \pi_{\lambda} \chi\left\|_{2}\left(\left\|V_{k}-V\right\|_{L^{\infty}}+\left\|V_{k} R_{V_{k}} V_{k}-V R_{V} V\right\|_{L^{2} \rightarrow L^{2}}\right)\right\| \chi \pi_{-\lambda}^{t} \|_{2} .\right.
\end{aligned}
$$

As in Lemma 3.3 of [4], using the explicit Schwartz kernel of $\pi_{\lambda}$, one can see that if $|\lambda| \leq j$ there is a constant $C_{j}$ such that $\left\|\pi_{\lambda} \chi\right\|_{2} \leq C_{j}$ and $\left\|\chi \pi_{-\lambda}^{t}\right\|_{2} \leq C_{j}$. We need only show that $\left\|V_{k} R_{V_{k}} V_{k}-V R_{V} V\right\|_{L^{2} \rightarrow L^{2}} \rightarrow 0$ as $k \rightarrow \infty$. But since $\operatorname{Im} \lambda \geq 2 N+1 \geq 2 \max \left(\left\|V_{k}\right\|_{L^{\infty}},\|V\|_{L^{\infty}}\right)+1$, the operators $R_{V_{k}}(\lambda)$ and $R_{V}(\lambda)$ are holomorphic functions of $\lambda$, with norms that are uniformly bounded in this region. Since

$$
R_{V_{k}}(\lambda)-R_{V}(\lambda)=R_{V_{k}}(\lambda)\left(V-V_{k}\right) R_{V}(\lambda),
$$

$\left\|R_{V_{k}}(\lambda)-R_{V}(\lambda)\right\| \rightarrow 0$ as $k \rightarrow \infty$. Thus $\left\|V_{k} R_{V_{k}} V_{k}-V R_{V} V\right\|_{L^{2} \rightarrow L^{2}} \rightarrow 0$ as $k \rightarrow \infty$.

A similar argument shows that $\left\|I-S_{V_{k}}(\lambda)\right\|_{1}$ and $\left\|I-S_{V}(\lambda)\right\|_{1}$ are bounded uniformly for $\operatorname{Im} \lambda \geq 2 N+1,|\lambda| \leq j$. Using (6), we see that $\operatorname{det} S_{V_{k}}(\lambda) \rightarrow$ $\operatorname{det} S_{V}(\lambda)$ and thus

$$
\log \left|\operatorname{det} S_{V}(\lambda)\right| \leq M|\lambda|^{q} \text { if } \operatorname{Im} \lambda \geq 2 N+1 \text { and }|\lambda| \leq j .
$$


In the next step, we characterize those $V \in L_{\text {comp }}^{\infty}(K ; F)$ for which the order of growth of the resonance counting function is strictly less than the dimension $d$. For $N, M, q>0$, let

$$
B(N, M, q)=\bigcap_{j \geq 2 N+1} A(N, M, q, j)
$$

Note that $B(N, M, q)$ is closed by Lemma 2.1 .

Lemma 2.2. Let $V \in L^{\infty}(K ; F)$, with

$$
\lim \sup _{r \rightarrow \infty} \frac{\log N_{V}(r)}{\log r}<d .
$$

Then there exist $N, M, l \in \mathbb{N}$ such that $V \in B(N, M, d-1 / l)$.

Proof. By [2, Lemma 4.2], there is a $p<d$ such that

$$
\lim \sup _{r \rightarrow \infty} \frac{\log \max _{0<\theta<\pi} \log \left|\operatorname{det} S_{V}\left(\left(2\|V\|_{L^{\infty}}+1\right) i+r e^{i \theta}\right)\right|}{\log r}=p .
$$

In fact, the continuity of $\operatorname{det} S_{V}(\lambda)$ in this region implies that this bound is true for $0 \leq \theta \leq \pi$. It follows that there is a $p^{\prime} \geq p, p^{\prime}<d$, and an $M \in \mathbb{N}$ such that

$$
\log \left|\operatorname{det} S_{V}(\lambda)\right| \leq M|\lambda|^{p^{\prime}}
$$

when $\operatorname{Im} \lambda \geq 2\|V\|_{\infty}+1$. Choose $l \in \mathbb{N}$ so that $p^{\prime} \leq d-1 / l$ and $N \in \mathbb{N}$ so that $N \geq\|V\|_{\infty}$, and then $V \in B(N, M, d-1 / l)$ as desired.

Lemma 2.3. The set

$$
\mathcal{M}=\left\{V \in L_{\mathrm{comp}}^{\infty}(K ; F): \lim \sup _{r \rightarrow \infty} \frac{\log N_{V}(r)}{\log r}=d\right\}
$$

is $a G_{\delta}$ set.

Proof. By Lemma 2.2, the complement of $\mathcal{M}$ is contained in

$$
\bigcup_{(N, M, l) \in \mathbb{N}^{3}} B(N, M, d-1 / l)
$$

which is an $F_{\sigma}$ set since it is a countable union of closed sets. By [2, Lemma $4.2]$, if $V \in \mathcal{M}$, then $V \notin B(N, M, d-1 / l)$ for any $N, M, l \in \mathbb{N}$. Thus

$$
\mathcal{M}^{c}=\bigcup_{(N, M, l) \in \mathbb{N}^{3}} B(N, M, d-1 / l)
$$

and $\mathcal{M}$ is the complement of an $F_{\sigma}$ set.

We can now prove our theorem.

Proof of Theorem 1.1. Since Lemma 2.3 shows that $\mathcal{M}$ is a $G_{\delta}$ set, we need only show that $\mathcal{M}$ is dense in $L^{\infty}(K ; F)$. To do this, we use a slight modification of the proof of $[2$, Corollary 1.3]. We give the proof here for the convenience of the reader. Let $V_{0} \in L^{\infty}(K ; F)$ and let $\epsilon>0$. By [20, Theorem 2], we may choose a spherically symmetric $V_{1} \in L^{\infty}(K ; \mathbb{R})$ so that $V_{1} \in \mathcal{M}$. We now consider the 
function $V(z) \equiv V(z, x)=z V_{1}(x)+(1-z) V_{0}(x)$. This potential satisfies the conditions of $\left[2\right.$, Theorem 1.1], with $V(0)=V_{0}$ and $V(1)=V_{1}$. Thus, by $[2$, Theorem 1.1], there exists a pluripolar set $E \subset \mathbb{C}$, so that for $z \in \mathbb{C} \backslash E$, we have

$$
\lim \sup _{r \rightarrow \infty} \frac{\log N_{V(z)}(r)}{\log r}=d .
$$

In particular, since $E \uparrow \mathbb{R} \subset \mathbb{R}$ has Lebesgue measure 0 (e.g. [7, Section 12.2]), we may choose a point $z_{0} \in \mathbb{R}, z_{0} \notin E$, with $\left|z_{0}\right|<\epsilon /\left(1+\left\|V_{0}\right\|_{L^{\infty}}+\left\|V_{1}\right\|_{L^{\infty}}\right)$. Then $V\left(z_{0}\right) \in \mathcal{M}$ and $\left\|V\left(z_{0}\right)-V_{0}\right\|_{L^{\infty}}<\epsilon$. Note that if $V_{0}$ is real-valued (respectively, complex-valued) then so is $V\left(z_{0}\right)$.

\section{References}

[1] T. Christiansen, Schrödinger operators with complex-valued potentials and no resonances. To appear, Duke Math. Journal.

[2] _ Several complex variables and the distribution of resonances for potential scattering. To appear, Commun. Math. Phys.

[3] R. Froese, Asymptotic distribution of resonances in one dimension, J. Differential Equations 137 (1997), 251-272.

[4] _ Upper bounds for the resonance counting function of Schrödinger operators in odd dimensions, Canad. J. Math. 50 (1998), no. 3, 538-546.

[5] R. Melrose, Polynomial bound on the number of scattering poles, J. Funct. Anal. 53 (1983), 287-303.

[6] _ Geometric Scattering Theory, Cambridge University Press, 1995.

[7] T. Ransford, Potential theory in the complex plane, Cambridge University Press, Cambridge, 1995.

[8] A. Sá Barreto, Remarks on the distribution of resonances in odd dimensional Euclidean scattering, Asymptot. Anal. 27 (2000), 161-170.

[9] _ M. Zworski, Existence of resonances in three dimensions, Comm. Math. Phys. 49 (1995), 401-415.

[10] _ M. Zworski, Existence of resonances in potential scattering, Comm. Pure Appl. Math. 49 (1996), no. 12, 1271-1280.

[11] B. Simon, Operators with singular continuous spectrum: I. general operators, Anal. Math. 141 (1995), 131-145.

[12] $ـ$ Resonances in one dimension and Fredholm determinants, J. Funct. Anal. 178 (2000), 396-420.

[13] Trace Ideals and their Applications, London Mathematical Society Lecture Note Series 35, Cambridge University Press, 1979.

[14] P. Stefanov, Sharp upper bounds on the number of the scattering poles. To appear, J. Funct. Anal.

[15] G. Vodev, Sharp polynomial bounds on the number of scattering poles for perturbations of the Laplacian, Comm. Math. Phys. 146 (1992), 205-216.

[16] M. I. Voitsekhovskii, "Generic set," Encyclopaedia of Mathematics, Kluwer Academic Publishers, Dordrecht, 1989.

[17] D. Yafaev, Mathematical Scattering Theory, Translations of Mathematical Monographs 105, American Mathematical Society, Providence, RI, 1992.

[18] M. Zworski, Poisson formulae for resonances, Séminaire sur les Équations aux Dérivées Partielles, 1996-1997, Exp. No. XIII, 14pp., École Polytech., Palaiseau, 1997.

[19] _ Counting scattering poles. In: Spectral and scattering theory (Sanda, 1992), 301-331, Lecture Notes in Pure and Appl. Math. 161, New York: Dekker, 1994.

[20] Sharp polynomial bounds on the number of scattering poles of radial potentials, J. Funct. Anal. 82 (1989), 370-403. 
[21] Sharp polynomial bounds on the number of scattering poles, Duke Math. J. 59 (1989), no. 2, 311-323.

[22] , Distribution of poles for scattering on the real line, J. Funct. Anal. 73 (1987), $277-296$.

Department of Mathematics, University of Missouri, Columbia, Missouri 65211

E-mail address: tjc@math.missouri.edu

Department of Mathematics, University of Kentucky, Lexington, KY 40506-0027

E-mail address: hislop@ms.uky.edu 